\title{
Editorial: Modelling and computational challenges in granular materials
}

\author{
T. Weinhart ${ }^{1}$ - A. R. Thornton ${ }^{1,2}$ - I. Einav $^{3}$
}

Published online: 25 November 2015

(C) OWZ 2015

Granular materials - conglomerates of macroscopic particles - are challenging systems to model. It is simply too costly to simulate the huge amount of particles in most systems in full detail. Even most intricate simulation methods, such as discrete particle simulations for modelling the full particle dynamics and direct numerical simulations to model a fluid flowing through the granular material, need to simplify particle properties, contact models and boundary conditions to become computationally feasible. Continuum methods efficiently simulate the bulk dynamics, based on simplified constitutive models that represent the granular materials efficiently, as effective media. However, the success of general constitutive relationships is still limited, because the inherent spatial and temporal scales of the particle dynamics are not well separated from the relevant macroscopic scales. Thus, the available constitutive models are based on simplifying assumptions that limit their range of applicability.

The challenge for the modeller is therefore to choose the right method and scale to simulate a material efficiently and accurately: Is it better to use a discrete particle simulation, a probabilistic method, or a continuum approach? How can the results of different methods be compared with each other

T. Weinhart

t.weinhart@utwente.nl

A. R. Thornton

a.r.thornton@utwente.nl

I. Einav

itai.einav@sydney.edu.au

1 Multiscale Mechanics, CTW/MESA+, University of Twente, Enschede, The Netherlands

2 Mathematics of Computational Science, EWI/MESA+, University of Twente, Enschede, The Netherlands

3 School of Civil Engineering, The University of Sydney, Sydney, Australia and with experiments? And, since it is impossible to simulate the full physics of the material, the question arises: which parameters control the macroscopic behaviour, and which can be safely ignored? Additionally, many models only have a limited range of applicability, especially because it is not obvious how to simulate particles in transition regions. The papers in this special issue consider these questions.

We start with two papers that use particle simulations to validate continuum approaches. These papers explore the range of applicability of the well-known $\mathrm{m}(\mathrm{I})$ rheology for inertial flows. The first contribution by Kneib, Faug, Dufour and Naaim uses the Discrete Element Method (DEM) to show that the inertial rheology can predict the force experienced by obstacles subjected to granular flows; the second paper by Ries, Brendel, and Wolf describes how transient states in simple shear flows can be modelled using the Contact Dynamics (CD) method, extending the potential use of the $\mathrm{m}(\mathrm{I})$ rheology to dynamic situations and the initiation of flow. Both papers demonstrate that these intricate simulation methods can successfully validate the more efficient, but rather more simplified continuum models.

The following two papers question whether microscale simulations can provide quantitative agreement with real industrial and experimental systems. The first paper is a review by Windows-Yule, Tunuguntla, and Parker who present in detail when and how such comparisons can be made in flowing and vibrated systems. The paper by Rosato, Zuo, Blackmore, Wu, Horntrop, Parker, and Windows-Yule exemplifies this by presenting a comparison of experimental results, particle simulations, and an analytical model for a vibrated chain of particles.

Next, we explore micro-macro transition methods for granular mixtures. These methods map discrete particulate data to continuum fields, and are commonly used to validate and calibrate continuum models via particle simulations and 
experimental data. Tunuguntla, Thornton, and Weinhart extend the coarse-graining micro-macro method to multicomponent granular flows and show how to extract local and dynamic continuum fields from discrete particle data. A similar approach is applied by Staron and Philips to a set of discrete particle simulations of bidispersed granular mixtures. The resulting continuum variables-partial stresses and densities-are then used to predict how the flow rheology is influenced by the existence of two grain sizes.

Finally, we compare different simulational techniques for porous and fluidised granular beds. The contribution by Müller, Third, and Chen compares direct numerical simulations (Lattice-Boltzmann method, LBM) of the flow through a fluidised bed with continuum Computational Fluid Dynamics simulations, reporting significant differences between the two methods.

Some common conclusions can be drawn from the contributions to this special issue: Because direct numerical simulations of large granular systems in their full complexity are not feasible, simplifying assumptions have to be made. Many different modelling approaches are shown here, which approximate the behaviour of the granular material with a varying degree of complexity. The more detailed discrete particle simulations (DEM and CD) and probabilistic methods (LBM) capture the relevant physics well but are so complex that only small systems can be simulated. Continuum models, discretised by Finite Element or Finite Volume approaches, have less degrees of freedom and can therefore simulate the behaviour of large systems, but have a limited range of applicability. The continuing challenge for the field is to extend the accuracy and range of applicability of the continuum methods, validate them by microscopic simulations, analysis, and experiments, and to find good (microscopic) simulational techniques where continuum methods cannot be used reliably.

The fundamental goal may be to find a comprehensive model for granular materials whose range of applicability is as wide as possible. However, one may also seek the inverse goal: to define the simplest yet adequate models for various classes of situations. Achieving either goal will require robust constitutive relations, that take into account relevant microscopic scales. Obtaining such relations may require a multiscale approach, where both discrete (micro) and continuum (macro) models are used in conjunction. This can be done in a coupled or decoupled fashion. In a decoupled approach, a continuum model is validated by discrete simulations a priori, and then applied to the whole domain. In the coupled approach, discrete and continuum simulations are run in parallel and coupled either spatially (i.e. the discrete simulations are applied to parts of the domain where the continuum method fails) or homogeneously (i.e. the discrete simulations are used to obtain closure relations for the continuum model). Either way, the challenge of choosing the right method for a given situation remains, which will keep granulists busy for the time to come. 